\title{
An Experimental Study of the Reduplication of Gradable Adjectives in Mandarin Chinese
}

\author{
Heather Pan \\ Big Bridge Academy, Wuxi, China \\ Email: heather.my.pan@outlook.com
}

How to cite this paper: Pan, H. (2020). An Experimental Study of the Reduplication of Gradable Adjectives in Mandarin Chinese. Open Journal of Social Sciences, 8, 447-452. https://doi.org/10.4236/jss.2020.88037

Received: June 15, 2020

Accepted: August 28, 2020

Published: August 31, 2020

\begin{abstract}
Compared to English, Mandarin Chinese has a rich inventory of reduplicated forms of adjectives. It has been argued that reduplicated forms of gradable adjectives convey an additional inference that the speaker has the relevant direct perceptual experience (Tang, 1988; Zhu, 2003; Liu, 2013). This article investigates whether the reduplicated form has this additional perceptual inference for native speakers by experimental studies. Our experiment results show that the reduplicated form of scalable adjectives does not require the perceptual inference compared to its base form, contra the previous studies.
\end{abstract}

\section{Keywords}

Gradable Adjective, Reduplication, Perceptual Inference, Mandarin Chinese

\section{Introduction}

In Mandarin Chinese, typical scalar adjectives such as gao "tall", ai "short", pang "fat" behave quite differently from its counterparts in English, and probably many other languages in the world. One long-term puzzle is that when a scalar adjective is the predicate of the sentence, its bare form cannot give rise to the positive interpretation, but only a comparative reading (Sybesma, 1999; Huang, 2006; Gu, 2008; Liu, 2010a; Liu, 2010b; Grano, 2012; Liu, 2018), as in (1).

1) Yuehan gao

John tall

"John is taller" But cannot be "John is tall"

To express a meaning like "John is tall", at least two ways are possible. One typical way is to add an unstressed, intuitively vacuous degree adverb hen "very", as in (2).

2) Yuehan hen gao 
John very tall

"John is tall"

Another option to convey the positive interpretation, which is the main focus of this paper, is to use its reduplicated form. For instance, a monosyllabic gradable adjective $\mathrm{A}$ is reduplicated as AA-de as in (3), in which the particle de is usually obligatory and is considered as the byproduct of reduplication (Law, 2006).

(3) Yuehan gao-gao-de

John tall-tall-DE

"John is tall $\mathrm{RED}_{\mathrm{RE}}$ "

While the literature has been assuming that both (2) and (3) can convey the positive meaning "John is tall", it has also been noticed that the two are not exactly equivalent. In particular, the reduplicated form often carries some extra flavor besides the positive degree when used by the speaker. The problem is that such an extra flavor is vague and intuitive, but hard to be paraphrased and formalized. For instance, as summarized in Liu (2013), most previous proposals (Wang, 1943; Zhu, 1982; Zhu, 1980; Lu et al., 1980; Zhu, 2003; Liu et al., 2004) argue that the reduplicated form in (3) denotes some life-like or vivid state of being tall, and the degree of John's tallness should not be too high or too high, but a "appropriate" one.

This paper investigates what the exact meaning of the reduplicated form of adjectives in Mandarin Chinese is and under what conditions people will use the reduplicated form. In particular, we are interested in a claim in Liu (2013), based on Tang (1988) and Zhu (2003), such that the reduplicated form of a gradable adjective conveys an extra inference that the speaker has a direct perceptual experience. Illustrating with the examples (2) and (3), Liu (2013) proposes that the use of the reduplicated form as in (3) implies that the speaker has direct visual experience concerning John's height. In other words, the speaker must have in fact seen John before to get to the conclusion that John is tall. While to utter (2), the speaker doesn't need to have such direct perceptual experience, and indirect experience like knowing the height of John via his record is sufficient.

While Liu's claim that the reduplicated form of gradable adjectives conveys the perceptual inference is intuitively correct, it is still based on his introspective judgements on a limited set of reduplicated gradable adjectives. There is barely any empirical study showing that such a perceptual inference is actually obligatorily conveyed or just arises as an implicature or preference that can be potentially canceled. In order to solve the problem, we carry out an experiment on native speakers of Mandarin in terms of whether the perceptual inference obligatory exists for a reduplicated adjective.

Our experiment is designed to investigate whether the reduplicated form carries an extra perceptual inference compared to its base form (with the semantically vacuous degree adverb hen). The main idea behind the experimental design is that, if Liu's claim is correct, then we predict that when someone without the relevant direct perceptual experience utters the sentence involving the redupli- 
cated form, the addressee should judge it as infelicitous, or unnatural. On the other hand, no such unnaturalness will arise if the base form is uttered in such a scenario. Our results of the experiment fail to reject the null hypothesis that the reduplicated form of gradable adjectives does not entail the perceptual inference and the relationship between base form and AA-form does not depend on whether the scenario is with perception or without perception, which reject Liu's (2013) claim. The experimental study makes a novel contribution to the current field by demonstrating that subtle changes in expressions could deliver different messages and evoke various emotions and responses, and answers questions in cultural differences and communication, in addition to its potential value in the study of linguistics.

\section{The Experiment}

The experiment is intended to investigate whether there is a difference regarding the perceptual inference between the base form of gradable adjective and its reduplicated form. In each trial, the participants saw a paragraph describing a certain scenario, which either entails the speaker of the target sentence has the relevant perceptual experience (WP) or does not (OP). Then the participants are asked to give the acceptability ratings in terms of how natural it is for the speaker to utter the target sentence, either with its base form (BASE) or with its reduplicated form (RED), in that scenario. The rating is calculated in each condition. There are two possible hypotheses. According to the experimental hypothesis, the reduplicated form of gradable adjectives additionally entails the perceptual inference compared to its base form, thus predicting the use of the reduplicated form of gradable adjectives is significantly degraded in scenarios lacking the perceptual experience (OP) compared to its use in scenarios involving the perceptual experience (WP), while its base form can be naturally used in both kinds of scenarios. In contrast, the null hypothesis, which holds that the reduplicated form of gradable adjectives does not entail the perceptual inference, predicts that the use of the reduplicated form of gradable adjectives should be fine in both kinds of scenarios, just like its base form. The goal of an experimental study is to investigate how some element or elements, the independent variable, affect some behavior or outcome, the dependent variable. In this experiment, one of the independent variables is the adjectival predicate with two levels: base form and reduplicated form. The other independent variable is the scenario with two levels: with perception or without perception.

\subsection{Methods}

Subjects. 59 male and female participants, whose ages ranging from 10 to 44, are collected from Zhejiang Province in China. All subjects speak Mandarin Chinese as their native language.

Materials. It is a $2 \times 2$ within-subjects design, with 4 conditions. The dependent variable of the experiment is the acceptability ratings, and the 2 independent variables are the adjectival predicate (Base form or Reduplicated form) and 
the scenario (With perception or Without perception), resulting in the following 4 conditions: BASE_WP (the use of base form in a scenario with perceptual experience), BASE_OP (the use of base from in a scenario without perceptual experience), RED_WP (the use of reduplicated form in a scenario with perceptual experience), and RED_OP (the use of reduplicated form in a scenario without perceptual experience). We selected four gradable adjectives pang "fat", shou "thin", $k u$ "bitter", tian "sweet" for use in the experiment, which creates 16 test trials in total. The utterance of those gradable adjectives are carefully manipulated to ensure all scenarios are pragmatically reasonable and easily imaginable. All materials are in Chinese. A sample stimulus is given in (5)-(8).

5) Scenario WP (with perceptual experience)

Flora tried a new drink in the shop. Her friend asks how the coffee tastes. Flora says that the coffee is sweet.

6) Scenario OP (without perceptual experience)

Flora saw a new drink in the shop. Her friend asks how the coffee tastes. Flora hasn't tried the new coffee yet. She saw the lists of ingredients on the bottle. She thinks it contains a lot of sugar, so she says that the coffee is sweet.

7) Sentence involving the base form of gradable adjectives (BASE)

zhe kafei hen tian.

This coffee very sweet.

"This coffee is very sweet."

(8) Sentence involving the base form of gradable adjectives (RED)

Zhe kafei tian tian de

This coffee sweet sweet de.

"This coffee is sweet RED. $_{\text {. }}$

Procedure. The materials of the experiment were created in Tengxun Wenjuan in the form of questionnaires and a link to the questionnaire was distributed to each participant via smart phones. In each trial of the experiment, the subject is shown a paragraph describing a specific scenario, then they were directed to grade the degree of naturalness of the sentence including a relevant adjectival form relative to that particular scenario from least natural to most natural on a scale of 1 to 5 . We tested 59 subjects on all conditions in order to control the potential individual variance since we are comparing the ratings of the target sentence between scenarios WP and scenarios OP respectively. Each subject completed a set of 16 trials ( 4 adjectives, each associated with 4 conditions). Before the experiment subjects were not told about the real purpose of the experiment and most of them did not tend to guess the aim of the experiment.

\subsection{Results and Discussions}

We collected 59 subjects and used 39 data from them. Among the 16 questions we presented to the subjects, question 1 and 15 are the most natural ones. So we didn't use the subject's data who graded these questions lower than 3 . We put all the accepted data into one form that is divided into four categories and performed a two way repeated measures ANOVA analysis on the data set. 
We performed a two-factor ANOVA with reduplications in Excel (Version 16.0.4266.1003) on the data set. The results are shown in Figure 1. We find that the effect of the factor Adj-Form $(\mathrm{F}(1,620)=0.021, p>0.05)$ is not significant while the effect of the factor Scenario $(\mathrm{F}(1,620)=74.13, p<0.005)$ is significant. Crucially, the effect of the interaction between the two factors $(\mathrm{F}(1,620)=0.005$, $p>0.5)$ is not significant. The results show that the scenario has a significant effect for both the base form and the reduplicated form, and based on the average we see that the utterance of both forms become degraded when the speaker does not have any perceptual experience. Crucially, since the interaction of adjectival forms and scenarios is not statistically significant, we fail to reject the null hypothesis and the relationship between base form and AA-form does not depend on whether the scenario is with perception or without perception. In other words, our studies show that Liu (2013)'s claim that the reduplicated form of a gradable adjective conveys an extra inference that the speaker has the relevant direct perceptual experience is not correct.

\section{General Discussion}

This article explores the exact meaning of adjective reduplication in Chinese, and under what circumstances people will use it. We are interested in a claim by Liu (2013) that reduplicated forms of graded adjectives convey an additional inference that the speaker has direct perceptual experience.

In order to solve the problem that perceptual inference is mandatory, we conducted an experiment on whether there is necessary perceptual reasoning for adjectival reduplication for Chinese native speakers.

The experiment investigates whether the reduplicated form has additional perceptual inference, compared with its basic form. Our experiment results did

\begin{tabular}{|c|c|c|c|c|c|c|}
\hline ANOVA: Two-Factor with Repli & & & & & & \\
\hline SUMMARY & with perception & without perception & Total & & & \\
\hline \multicolumn{7}{|c|}{ base } \\
\hline Count & 156 & 156 & 312 & & & \\
\hline Sum & 637 & 519 & 1156 & & & \\
\hline Average & 4.083333333 & 3.326923077 & 3.705128205 & & & \\
\hline Variance & 0.928494624 & 1.356947891 & 1.282545964 & & & \\
\hline \multicolumn{7}{|c|}{ reduplicate } \\
\hline$\overline{\text { Count }}$ & 156 & 156 & 312 & & & \\
\hline Sum & 640 & 520 & 1160 & & & \\
\hline Average & 4.102564103 & 3.333333333 & 3.717948718 & & & \\
\hline Variance & 1.099090157 & 1.513978495 & 1.450737901 & & & \\
\hline \multicolumn{7}{|c|}{ Total } \\
\hline Count & 312 & 312 & & & & \\
\hline Sum & 1277 & 1039 & & & & \\
\hline Average & 4.092948718 & 3.330128205 & & & & \\
\hline Variance & 1.010625361 & 1.430857861 & & & & \\
\hline & & & & & & \\
\hline \multicolumn{7}{|l|}{ ANOVA } \\
\hline Source of Variation & SS & $\mathrm{df}$ & MS & F & $\mathrm{P}$-value & F crit \\
\hline Adj. Form & 0.025641026 & 1 & 0.025641026 & 0.020937811 & 0.884995523 & 3.856500942 \\
\hline Scenario & 90.77564103 & 1 & 90.77564103 & 74.12508654 & $6.04468 \mathrm{E}-17$ & 3.856500942 \\
\hline Interaction & 0.006410256 & 1 & 0.006410256 & 0.005234453 & 0.942347091 & 3.856500942 \\
\hline Within & 759.2692308 & 620 & 1.224627792 & & & \\
\hline Total & 850.0769231 & 623 & & & & \\
\hline
\end{tabular}

Figure 1. The results of the two-factor ANOVA with reduplication on the data set. 
not reject the reduplicated form of scalable adjectives that do not require perceptual inference, and the relationship between the basic form and AA-form does not depend on whether the situation is perceptual, and thus Liu's (2013) claim is rejected.

\section{Conflicts of Interest}

The author declares no conflicts of interest regarding the publication of this paper.

\section{References}

Grano, T. (2012). Mandarin Hen and Universal Markedness in Gradable Adjectives. Natural Language \& Linguistic Theory, 30, 413-565. https://doi.org/10.1007/s11049-011-9161-1

Gu, Y. (2008). Studies of Tense, Aspect and Chinese Timereference. In Y. Shen, \& S. L. Feng (Eds.), Contemporary Linguistic Theories and Related Studies of Chinese (pp. 97-117). Beijing: Shangwu Yinshuguan.

Huang, S.-Z. (2006). Property Theory, Adjectives and Modification in CHINESE. Journal of East Asian Linguistics, 15, 343-369. https://doi.org/10.1007/s10831-006-9002-0

Law, P. (2006). Adverbs in A-Not-A questions in Mandarin Chinese. Journal of East Asian Linguistics, 15, 97-136. https://doi.org/10.1007/s10831-005-4916-5

Liu, C.-S. L. (2010a). The Positive Morpheme in Chinese and the Adjectival Structure. Lingua, 120, 1010-1056. https://doi.org/10.1016/j.lingua.2009.06.001

Liu, C.-S. L. (2010b). The Chinese Gèng Clausal Comparative. Lingua, 120, 1579-1606. https://doi.org/10.1016/j.lingua.2009.09.005

Liu, C.-S. L. (2013). Reduplication of Adjectives in Chinese: A Default State. Journal of East Asian Linguistics, 22,101-132. https://doi.org/10.1007/s10831-012-9099-2

Liu, C.S.L. (2018). Projecting adjectives in Chinese. Journal of East Asian Linguistics, 27, 67-109. https://doi.org/10.1007/s10831-018-9166-4

Liu, Y.-H., Pan, W.-Y., \& Gu, W. (2004). Modern Chinese Grammar. Beijing: Shangwu Yinshuguan.

Lu, S.-X. et al. (1980). Eight Hundred Words in Modern Chinese. Beijing: Shangwu Yinshuguan.

Sybesma, R. (1999). The Mandarin VP. Dordrecht: Kluwer. https://doi.org/10.1007/978-94-015-9163-8

Tang, T-C. (1988). Studies on Chinese Morphology and Syntax. Taipei: Student Book Co., Ltd.

Wang, L. (1943). Modern Chinese Grammar. Beijing: Shangwu Yinshuguan.

Zhu, D.-X. (1980). Studies on Syntax of Modern Chinese. Beijing: Shangwu Yinshuguan.

Zhu, D-X. (1982). Lectures on Chinese Syntax. Beijing: Shangwu Yinshuguan.

Zhu, J.-S. (2003). The Syntax and Semantics of Reduplicated Adjectives. Yuwen Yanjiu $88,9-17$. 